\title{
Adaptive ECG Compression Using Support Vector Machine
}

\author{
Sándor M. Szilágyi ${ }^{1}$, László Szilágyi ${ }^{1,2}$, and Zoltán Benyó \\ ${ }^{1}$ Sapientia - Hungarian Science University of Transylvania, \\ Faculty of Technical and Human Science, Târgu-Mureş, Romania \\ szs@ms.sapientia.ro \\ ${ }^{2}$ Budapest University of Technology and Economics, \\ Dept. of Control Engineering and Information Technology, Budapest, Hungary
}

\begin{abstract}
An adaptive, support vector machine based ECG processing and compression method is presented in this study. The conventional pre-filtering algorithm is followed by a characteristic waves (QRS, T, $\mathrm{P})$ localization. The regressive model parameters that describe the recognized waveformes are determined adaptively using general codebook information and patient specific data. The correct regocnition ratio of the QRS waves was above 99.9\% using single channels from the MIT$\mathrm{BIH}$ database files. The adaptive filter properly eliminates the perturbing noises such as $50 / 60 \mathrm{~Hz}$ power line or abrupt baseline shift or drift. The efficient signal coding algorithm can reduce the redundant data about 12 times. The good balance among proper signal quality for diagnosis and high compression rate is yielded by a support vector machine based system. The properly obtained wave locations and shapes, using a high compression rate, can form a solid base to improve the diagnosis performance in clinical environment.
\end{abstract}

Keywords: signal compression, QRS clustering, support vector machine, adaptive estimation.

\section{Introduction}

Nowadays the computerized ECG signal processing can be considered a welldeveloped application. An on-line performing analyzer and encoder system must be able to evaluate the signal with maximum few seconds delay to recognize in time the potentially dangerous and life threatening arrhythmia. These systems are based on filtering, beat detection (recognition and clustering), classification, storage and diagnosis.

Despite the presence of serious perturbation, a reliable analysis must involve at least the detection of $\mathrm{QRS}$ complexes, $\mathrm{T}$ and $\mathrm{P}$ waves, automatic rhythm analysis, classification and diagnosis, enabling physicians to derive more information for cardiac disease diagnosis. To perform a reliable evaluation of the ECG, it is important to correctly determine the position and amplitude of every characteristic event, as they play an important role at producing the diagnosis. The performance of an optimally functioning computerized ECG filtering algorithm

L. Rueda, D. Mery, and J. Kittler (Eds.): CIARP 2007, LNCS 4756, pp. 594 603, 2007.

(C) Springer-Verlag Berlin Heidelberg 2007 
is limited by the ability to separate the signal from artifacts, and by the amount and nature of distortion introduced by the filter. As a filter can perform much better using some predefined patient specific information, it is desired to use a post-filtering step to reduce the signal distortion. The speed and accuracy requirements during ECG processing represent a hard task, the varying shape of the ECG and the subjectivity of the diagnosis make it even harder.

The most important task in the ECG signal processing is the accurate localization of QRS complexes. The established QRS places constitute the basic a-priori information for all further processing steps. Many times the recorded ECG is disturbed by different kind of noises that can imply a pre-filtering or discarding of the studied segment. To assure the good quality of the ECG, and to prevent the loss of clinically significant information, the usage of human and artificial noise detection schemes is required. As the perturbing noise can only be diminished, but not eliminated from the measured signal, it is important to use processing methods with good noise susceptibility. The non-linear behavior of the human body requires adaptive processing that follows the patient's state.

The design of an optimal matched filter can increase the signal-to-noise ratio, but the non-stationary nature of the signal and noise in an ECG represents an obstacle in the application of these filters for QRS detection. A linear filter cannot properly whiten the non-linear ECG signal. Artificial neural networks (ANN) [12] are inherently non-linear models, so an ANN-based filtering is potentially useful. In practical use, the ANN model can adapt far better than linear models. The number of input units corresponds to the filter order that should not be increased too much, in order to assure constantly good transient properties. The selection of the right number of hidden layers is important to provide good learning speed and adaptation at the same time. After pre-processing, filtering, evaluation and model's parameter estimation, the signal reconstruction is needed. In this step, the post-filtering method knows the main ECG specific information, and can better separate all artificial noises. To create an efficient filter, it is necessary to use all ECG and patient dependent information. This problem can be handled only if the computer knows the formation of the ECG signal.

The collected noise during ECG signal acquisition makes almost meaningless the usage of loss-free compression [4. In this paper we focused on lossy methods as a compromise between bandwidth and final reconstruction possibility, using sophisticated medical knowledge-based reconstruction algorithms [9]. The signal's main characteristics are represented by exponential parameterization that is delivered by a processing system that uses support vector machine (SVM) [5]. This robust model involves the filtering, analysis and compression step of an automatic ECG evaluation method.

\section{Materials and Methods}

The proposed complex ECG signal compression algorithm can be divided into the following steps (see Fig. 10):

- Irregular signal recognition and processing; 


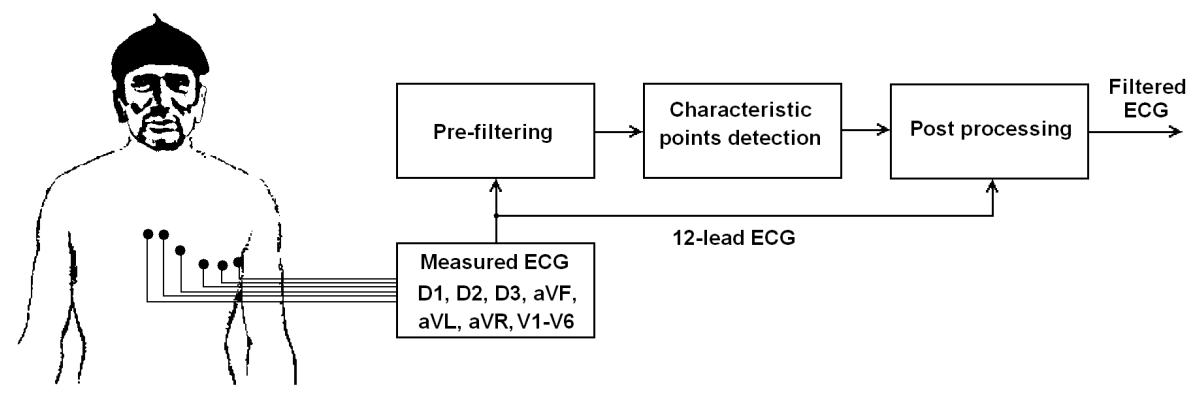

Fig. 1. The proposed ECG signal filtering, processing and compression

- Pre-filtering;

- Segmentation into R-R intervals, R-R period based filtering;

- Create/update a temporal template bank for whole beats;

- Determine all recognizable characteristic points (for R, T and P waves);

- R, T and $\mathrm{P}$ wave location based filtering;

- Extract the waveform estimation model's parameters;

- Post-filtering using pattern database and the model-based estimation;

- Complete the general and patient specific template bank for all recognized waves;

- Adjust long term prediction;

- Adaptive smoothing and advanced distortion analysis;

- Residual signal estimation, entropy coding, data storage, back-estimation;

- Medical parameter estimation and computer aided diagnosis.

Before starting the characteristic waveforms ( $\mathrm{R}, \mathrm{T}$ and $\mathrm{P}$ waves) recognition and proceed an accurate segmentation, we need to pre-filter the signal in order to eliminate all perturbing phenomena, like noise caused by the electrical network, the perturbing artifacts caused by bad contacts, motion or breathing. The elimination of the noise caused by the electrical network is recommended by windowed FFT and IFFT combined with a parameter estimator and filter, which contains the following steps:

- Perform a windowed FFT for an interval of length between five and twenty seconds. All intervals are overlapped by at least fifty percent of their length;

- Estimate the amplitude and phase of the 50 (60) $\mathrm{Hz}$ component and its harmonics from the amplitudes and phases of the adjacent frequencies;

- Modify the signal spectra;

- Process the IFFT algorithm;

- Performed a regressive elimination of the artificial noise caused by modifications in signal's spectra.

In our approach, because the studied signal has a non-linear behavior, we define a non-linear adaptive estimation algorithm. The main drawback of this 
method is the cumbersomeness to determine the optimal phase of the $50(60) \mathrm{Hz}$ component of the measured ECG signal, but paper [8] presents how to handle this problem.

In high frequency noise elimination, the non-linear parameter estimation methods could reach better performance than transformation methods.

Let $\hat{X}_{L}(n)$ and $\hat{X}_{R}(n)$ the $n$-th left and right aimed estimation, defined as:

$$
\begin{aligned}
& \hat{X}_{L}(n)=p_{L} \cdot \widetilde{X}_{L}(n)=p_{L} \sum_{i=-q}^{q} a_{L, i} X(n-i)+\left(1-p_{L}\right) \sum_{i=1}^{q} b_{L, i} \hat{X}_{L}(n-i) \\
& \hat{X}_{R}(n)=p_{R} \cdot \widetilde{X}_{R}(n)=p_{L} \sum_{i=-q}^{q} a_{R, i} X(n-i)+\left(1-p_{R}\right) \sum_{i=1}^{q} b_{R, i} \hat{X}_{R}(n-i)
\end{aligned}
$$

where $a_{L, i}, a_{R, i}, b_{L, i}$ and $b_{R, i}$ are prediction coefficients, $p_{L}$ and $p_{R}$ are balance probabilities determined by the dispersions

$$
\sigma_{\hat{X}_{L}-X}(n, l), \sigma_{\hat{X}_{R}-X}(n, l), \sigma_{\widetilde{X}_{L}-X}(n, l) \text { and } \sigma_{\widetilde{X}_{R}-X}(n, l),
$$

constrained by the condition $p_{L}+p_{R}=1$. For better separation of the signal from the noise, the length $l$ should select more than one R-R period. On-line processing requires the estimation to be delayed with at least $3 \cdot q$ samples, but preferably with more than one R-R interval, in order to minimize the differences of the efficiency between $\hat{X}_{L}(n)$ and $\hat{X}_{R}(n)$. The resulting sample $\hat{X}(n)$ is obtained by the following formula:

$$
\hat{X}(n)=p \sum_{i=-q}^{q} a_{i} \hat{X}_{L}(n-1)+(1-p) \sum_{i=-q}^{q} b_{i} \hat{X}_{R}(n-1) .
$$

The high level of the noise can massively degrade the performance of the parameter extraction models. In this situation, a well constructed transformation algorithm can outperform the parameter estimation procedure. Our proposed transformation method for QRS complex detection uses wavelets. The selected mother wavelet is:

$$
\Psi(t)=\frac{1}{\sqrt{2 \pi} \sigma} \cdot \exp \left(-\frac{t^{2}}{2 \sigma}\right) \cdot \sin (2 \pi \cdot \alpha \cdot t \cdot \exp (-\beta|t|))
$$

Parameters $\alpha$ and $\beta$ are selected according to the highest frequency in ideal (noise free) ECG signal, while $\sigma$ is the dispersion that acts like an attenuation factor for the wavelet's shape. After the analysis of more than 100 recordings, we obtained as a good robust result $\alpha=100$ and $\beta=1 / 3$. The robustness in this step is far more important, than a local performing index.

The first step in the recognition of the characteristic waveforms of the ECG consists of accurate detection of the QRS beats. These waveforms contain most of the signal's power, so they must be included into the template collection. This collection may be altered during ECG processing.

Although the automated waveform classification based on a decision tree algorithm could produce remarkable results, the self-organizing ( $\mathrm{SO}$ ) adaptive clustering [3] based method have several advantages: 
- It is not susceptible to variations of beat morphology and temporal characteristics;

- It can perform a real-time unsupervised learning;

- It needs much less amount of knowledge for the same performance.

The clusters are built up according to the following rules:

$-\sigma_{i} \leq \sigma_{\mathrm{Max}} ; \sigma_{\mathrm{Max}}$ is predetermined; $i=0 \ldots n$;

- The mean value of a cluster is $M_{i}$ and is determined in such a way, that $\sigma_{i}$ to be minimal;

- For every R ( $\mathrm{T}$ and $\mathrm{P}$ ) wave, which belongs to a cluster

$$
\|X\|=\sum_{i=0}^{n}\left(\frac{X_{i}-M_{i}}{\sigma_{i}}\right)^{2} \leq R_{\mathrm{Max}}
$$

where $R_{\text {Max }}$ is predetermined; $X$ is a vector, representing a wave in the space.

Each waveform in the template bank is represented by 8 characteristic points that were selected using a shape estimation error minimization process. The indicator vector of each waveform is represented as $\bar{X}^{T}=\left(p_{0}(X), \ldots, p_{n-1}(X)\right)$ where $n$ is the number of clusters and $p_{I}(X)$ is the probability that $X$ belongs to the cluster $C_{I}$, having the value:

$$
p_{I}(X)=\prod_{k=0}^{7} \frac{1}{\sigma_{I, k}} \exp \left(-\frac{\left(X_{k}-M_{I, k}\right)^{2}}{2 \sigma_{I, k}}\right) .
$$

The clustering process must work properly even if the studied patient manifests abnormal QRS wave patterns. To assure this, the main database must contain the most specific abnormal waveforms that are patient-free. It case of heavily patient dependent waveforms, such as ectopic beats, the studied waveforms are collected and included in a new, patient dependent cluster. These clusters are representative only for a patient but not for a larger group.

The optimal filter is based on the pre-processed signal and the template bank. Let

$$
\bar{X}(n)=\sum_{k=0}^{n r-1}\left(s_{k} \cdot \sum_{i=-q}^{q} a_{F, i} \cdot X_{k}(n-i)\right)
$$

and

$$
\widetilde{X}(n)=p_{F, X-\widetilde{X}}(n) \cdot \bar{X}(n)+\left(1-p_{F, X-\widetilde{X}}(n)\right) \cdot \sum_{i=-q}^{q} b_{i} B(m, i)
$$

be the processed data. The low value of $p_{F}, p_{F}<0.2$, justifies the need of the collection $B$, whose $m$-th element has the maximal correlation value with $\bar{X}(n)$.

The characteristic point localizer algorithm is performed in a similar way to the template bank building method. An important difference consists in the appliance manner of pre-filtered data. Firstly the template bank is created for 
every recognizable event. With the aid of pre-filtered data, we can minimize the isoelectric line displacement caused problem. Such a pre-filtered and denoised signal consist the entrance of a SVM trained ANN. This kind of formulation of learning leads to quadratic programming with linear constraints.

The problem of learning SVM [1] is formulated as a task of separating learning vectors $X_{i}$ into two classes of destination values $d=+1$ or $d=-1$ using maximal possible separation margin, that gives a high robustness to the obtained solution. The maximization task of function $Q(\alpha)$ is defined as follows:

$$
Q(\alpha)=\sum_{i=-p}^{p} \alpha_{i}-\frac{1}{2} \sum_{i=-p}^{p} \sum_{j=-p}^{p} \alpha_{i} \cdot \alpha_{j} \cdot d_{i} \cdot d_{j} \cdot K\left(x_{i} \cdot x_{j}\right)
$$

with linear constraints $\sum_{i=-p}^{p} \alpha_{i} \cdot d_{i}=0$, where $0 \leq \alpha_{i} \leq C$ [110]. The $\alpha$ values are Lagrange multipliers, and function $K$ represents the kernel, $p$ is the number of learning pairs and $C$ is a user defined constant (in our study $C$ was selected between 0.1 and 0.5 ). In this case we applied radial Gaussian kernel function. The output signal $y(x)$ of the SVM network in retrieval mode (after learning) is determined as the combination of kernels

$$
y(x)=\sum_{i=1}^{N_{S V}} \alpha_{S V i} \cdot d_{i} \cdot K\left(x_{S V i} \cdot x\right)+w_{\text {opt }}
$$

where $N_{S V}$ is the number of support vectors and $w_{o p t}$ is the optimal weight vector. Although SVM separates the data into two classes, the recognition of more ones is straightforward by applying either 'one against one' or 'one against all' methods [2]. After the ANN is trained, we used it to estimate the ECG as an output of a whitening filter.

The non-linear intermediate result is:

$$
Z_{p}(t)=f\left(\sum_{k=-j}^{j} c_{p k}(t) \cdot X(t+k)\right)
$$

where $X_{k}(t)=Y(t+k)$, and $f()$ is a normalized Gauss function. The $c_{p k}$ weight coefficients connect the input and the hidden layers. The output of the filter is:

$$
Y_{w}(t)=Y(t)-\hat{Y}(t)=Y(t)-\sum_{p=-i}^{i} c_{p}(t) \cdot Z_{p}(t) .
$$

The adaptive behavior of the filter is assured by the permanent variance of the genetic search method based upon least mean square (LMS) algorithm computed coefficients 4. Both the input signal and the selected template are processed through the main filter. During this process, the template bank is changing adaptively. The whitened template is:

$$
T_{w, r}(t)=T(r)-\sum_{p=-i}^{i} c_{p}(t) \cdot Z_{p}(t),
$$

where $r=j, \ldots, L-j$, and $L$ is the size of the template. The output of the matched filter will be: 


$$
Y_{w}(t)=\sum_{r=j}^{L-j} T_{w, r}(t) \cdot Y_{w}(t-L+r)
$$

After the signal is filtered, a smoothing operation should be performed to reduce the size of the compacted data. The compression strength should be selected in accordance with the diagnosis performance decrease from the recovered signal. The main aim of this algorithm is to decrease the length of the compressed signal and to keep the data quality as high as possible. Let

$$
\widetilde{X}_{[\mathrm{sm}]}(n)=\frac{1}{k}\left(\sum_{i=1}^{k-1} \widetilde{X}_{[\mathrm{sm}]}(n-i \tau)+\widetilde{X}(n)\right),
$$

where $k=2^{j}$, with $j$ and $\tau$ positive integers, and $\widetilde{X}(n)=Y_{w}(n)$. Normally the adjacent samples are highly correlated, and we select the positive integer $\tau$ that minimizes the auto-correlation function of the ECG signal. Usually the sampling delay $\tau$ is about half a QRS complex duration. The inverse transform is given by:

$$
\widetilde{X}(n)=k \cdot \widetilde{X}_{[\mathrm{sm}]}-\sum_{i=1}^{k-1} \widetilde{X}_{[\mathrm{sm}]}(n-i \tau) .
$$

In the meantime of the transform, the values of $\widetilde{X}(n)$ and $\widetilde{X}_{[\mathrm{sm}]}(n)$ can be modified with $k / 2$ in order to reduce the reconstruction error or the dispersion of the smoothed signal. The efficiency of this algorithm highly depends on the chosen values for $k$ and $\tau$.

Because the variance of the filtered and optionally smoothed signal $\sigma_{\widetilde{X}_{[\mathrm{sm}]}}(n, l)$ is too high to allow sufficient compression rate, a linear prediction transform is needed. This method eliminates the redundancy due to correlation between adjacent samples and beats. The resulting data

$$
Y(n)=P(n) \cdot \sum_{i=1}^{q} a_{E, i} \widetilde{X}(n-i)+(1-P(n)) \cdot \sum_{i=-q}^{q} b_{E, i} B(m, i),
$$

where $\left.P(n)=p_{E, \widetilde{X}_{[\mathrm{sm}]}-B(m)}(n)\right)$, support the calculation of the residual signal $r(n)=Y(n)-\widetilde{X}_{[\mathrm{sm}]}(n)$.

Verifying processes determine the compression caused performance decrease in accordance to square error and diagnostic robustness. More iterations should be calculated to determine the optimal set of parameters. In most cases, the estimation errors have nearly normal distribution. In order to reduce the length of the residual data, an adaptive method-based entropy coding is needed. For every moment we determine the dispersion $\sigma_{r}(n, l)$ and the probability $p_{\sigma_{r}(n, l)}(r(n, l))$ of the errors. The output value is obtained by:

$$
N_{[a c t]}(n, k)=I_{1}(n-k+1)+p_{1}(n-k+1) \cdot I_{2}(n-k+2)+\prod_{i=1}^{k-1} p_{i}(n-k+i) \cdot I_{k}(n)
$$

using $p_{i}(n-k+i)=p(r(n-k+i), l)$ and $I_{k-i}=\int_{-\infty}^{r(n-i)} p_{k-i}(n-i) d r$. 


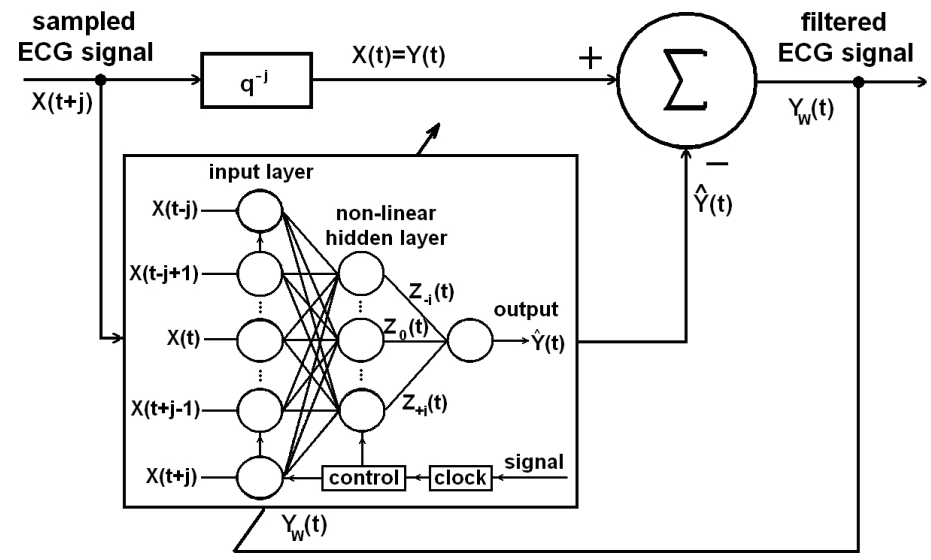

Fig. 2. The structure of the ANN-based adaptive filter

Table 1. Representation of the entropy coder's performance using 32 parameters

\begin{tabular}{llll}
\hline MIT-BIH record no. Theoretic entropy & Huffman code size & SVM-based coding \\
\hline 102 & 161677 & 193419 & 163589 \\
103 & 171223 & 201448 & 173295 \\
107 & 169442 & 200102 & 171142 \\
202 & 162735 & 192781 & 164814 \\
205 & 182493 & 214923 & 184456 \\
220 & 167321 & 198274 & 169121 \\
223 & 180039 & 211952 & 181935 \\
\hline
\end{tabular}

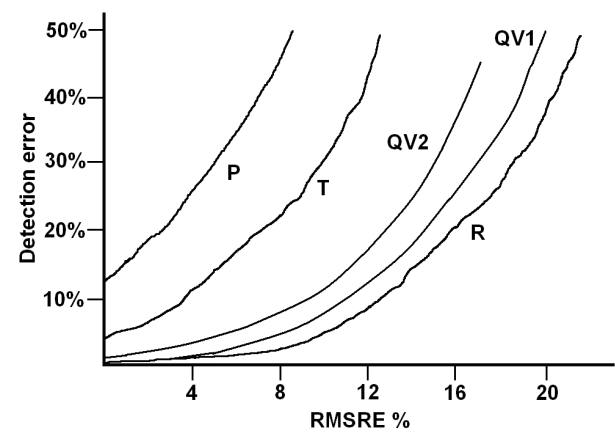

(a)

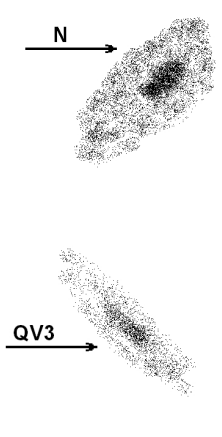

(b)

Fig. 3. (a) The recognition ratio of R, T, P and QV1, QV2 waves plotted against RMSRE; (b) Representation of various beat forms: the normal QRS beats are represented by $\mathrm{N}$, and three different ventricular extra beats are represented by QV1, QV2 and QV3 in plain representation (the calculation space has 32 dimensions, and the two most significant orthogonal combinations were selected for planar representation) 


\section{Results}

For a better comparability we used the MIT-BIH arrhythmia database for evaluation. All processing steps were tested on the ECG registrations of MIT-BIH database (sampled at $360 \mathrm{~Hz}$ with 11-bit resolution) and our own recordings (sampled at 200-500 Hz with 12-bit resolution). Most of these files contain one or two channels. The performance is determined by the number of estimation parameters, smoothing strength, resolution and sampling rate. The entropy coding can decrease at least 12 times the theoretical 'waste', compared with Huffman coding, during signal compacting.

The characteristic wave recognition performance heavily depends on the level of allowed root mean square error during signal compression. Figure 3 (a) represents the level of detection error of $\mathrm{P}, \mathrm{T}$ and $\mathrm{R}$ waves when the relative RMSE varies from 0 to $20 \%$. We investigated the detection error in function of the nature of $\mathrm{R}$ wave. The ventricular ectopic beats QV1 and QV2 included in this study were treated patient specific. Figure 3(b) illustrates the separation problem of various QRS waveforms in a 32-dimensional parameter space.

\section{Discussion and Conclusion}

Table 1 illustrates the compaction effectiveness for some of the most perturbed files, whose noise level and missed $\mathrm{R}$ wave detection rate was almost maximal. The new coding formula (19) has far better results than the adaptive Huffman coding. The elements distribution is close to normal and its change is not recommended without further knowledge. The smoothing strength should be adjusted by $k$ and $\tau$ (see (16), (17)). Experiments show that (Fig. 3) the $\mathrm{R}$ wave can be accurately recognized even if RMSRE is about $10 \%$. For $\mathrm{T}$ and $\mathrm{P}$ wave detection 7. the root mean square error must not exceed $3-5 \%$ of the signal's power. S (J), $\mathrm{Q}$ points and $\mathrm{U}$ wave cannot be recognized in most of the cases if RMSRE is higher than $1 \%$. The lower amplitude of the $\mathrm{T}$ and $\mathrm{P}$ waves is the main reason of the lower detection rate. The amplitude of the QV1 and QV2 ectopic beats is about the same as in case of $\mathrm{R}$ waves, but the uncommon patient-specific shape of them inhibit the usage of a priori waveforms (we considered new patients, so the database had not any information about them). It can be observed a smoother shape of the performance graph for the studied QV1 and QV2 ectopic beats caused by the much lower available occurrence (almost 100 times less).

An experimental real-time processing using this method needs a powerful computer able to perform massively parallel algorithms. In Holter telemetry and diagnostic systems [6], where a vast amount of data is acquired and transmitted by radio wave, the compression is an unavoidable step of the computerization. Sophisticated long computation and lingering unpack of the signal could be the major disadvantages of this process. Although quite often the calculation term doesn't admit on-line computerization (in multitasking mode), the rapid evolution of the computers will shortly change this fact. 
Acknowledgements. This research was supported by the Sapientia Institute for Research Programmes.

\section{References}

1. Burges, C.: A tutorial on support vector machines for pattern recognition. In: Fayyad, U. (ed.) Knowledge Discovery and Data Mining, pp. 1-43. Kluwer, Norwell, MA (2000)

2. Crammer, K., Singer, Y.: On the learnability and design of output codes for multiclass problems. In: Proc. 13th Conf. Comput. Learn Theor. pp. 35-46 (2000)

3. Lagerholm, M., Peterson, C., Braccini, G., Edenbrandt, L., Sörnmo, L.: Clustering ECG complexes using Hermite functions and self-organizing maps. IEEE Trans. Biomed. Eng. 47, 838-848 (2000)

4. Nave, G., Cohen, A.: ECG Compression Using Long-Term Prediction. IEEE Trans. Biomed. Eng. 40, 877-885 (1993)

5. Osowski, S., Hoai, L.T., Markiewicz, T.: Support vector machine-based expert system for reliable heartbeat recognition. IEEE Trans. Biomed. Eng. 51, 582-589 (2004)

6. Szilágyi, S.M.: Non-Linear Adaptive Prediction Based ECG Signal Filtering. In: Proc. 21st Ann. Int. Conf. IEEE EMBS, Atlanta, p. 286 (1999)

7. Szilágyi, S.M., Szilágyi, L.: Wavelet Transform and Neural-Network-Based Adaptive Filtering for QRS Detection. In: Proc. 22nd Ann. Int. Conf. IEEE EMBS, Chicago, pp. 1267-1270. Chicago (2000)

8. Szilágyi, S.M., Benyó, Z., Dávid, L.: Iterative ECG Filtering for Better Malfunction Recognition and Diagnosis. In: Proc. 5th IFAC Symp. Model. Contr. Biomed. Syst. Melbourne, pp. 295-300 (2003)

9. Szilágyi, S.M., Szilágyi, L., Benyó, Z.: ECG Signal Compression and Noise Distortion Effect Analysis. Proc. World Congr. Med. Phys. Biomed. Eng. Sydney, IFMBE Proc. 4, 4391 (2003)

10. Smola, A., Scholkopf, B.: A tutorial on support vector regression. Royal Holloway College, Univ. London, NeuroColt Tech. Rep. NV2-TR-1998-030

11. Vapnik, V.: Statistical learning theory. Wiley, New York (1998)

12. Xue, Q., Hu, Y.H., Tompkins, W.J.: Neural-Network-Based Adaptive Matched Filtering for QRS Detection. IEEE Trans. Biomed. Eng. 39, 317-329 (1992) 